\title{
Wide Baseline Matching using Triplet Vector Descriptor
}

\author{
Yasushi Kanazawa Koki Uemura \\ Department of Knowledge-based Information Engineering \\ Toyohashi University of Technology, Toyohashi 441-8580, JAPAN \\ kanazawa@tutkie.tut.ac.jp
}

\begin{abstract}
We propose an image matching method using triplet vector descriptor. The triplet vector descriptor consists of two different types of affine invariants: the gray level profile between two feature points and the two covariance matrices of those points. In order to establish point matches, we first vote the similarities of the triplet vector descriptors into candidate matches, and then, we verify the matches by normalized triangular region vectors, which are also affine invariant. After enforcing the uniqueness of the candidate matches, we finally adopt RANSAC with the epipolar constraint for removing outliers. By using our method, we can obtain correct matches on wide baseline matching problems. We show the effectiveness of our method by real image examples.
\end{abstract}

\section{Introduction}

Detecting correspondences between two or more images is a very important task and the first step for many computer vision applications. So, many methods have been proposed in the past $[5,6,7,10,12,13,14]$.

There are three types in image matching: point-based, line-segment-based, and region-based. The point-based matching first detects feature points in each image by an interest point detector, such as Harris operator [2] and SUSAN [11], and then makes correspondences from point pairs [5, 14]. But, this type of matching cannot be apply to wide baseline cases, because it is based on correlations between local regions centered on the feature points and the correlations are seriously affected by positions of view points. The line-segment-based matching also detects feature points in each image or detect edges directly, and makes correspondences from line segment pairs by affine invariants $[1,12]$ or geometrical constraints. The region-based matching usually uses affine invariant on planes in a scene $[7,9,10,13]$. Since the line-segment-based and the region-based matchings usually use affine invariant, they can apply to wide baseline cases. But, in the method based on these matching principles, we need additional procedures for detect features [7] or need to adopt geometrical hashing [1].

In this paper, we propose an image matching method using the triplet vector descriptor. It is an affine invariant on planes and consists of the gray level profile between two feature points and the two covariance matrices of those points. In order to detect correspondences, we first vote the similarities of the triplet vector descriptors into candidate matches, we then verify the matches with high similarities by another affine invariant: 
normalized triangular region vectors. After enforcing the uniqueness of the candidate matches, we finally adopt RANSAC with the epipolar constraint for removing outliers. By using our method, we can obtain correct matches on wide baseline matching problems. We show the effectiveness of our method by real image examples.

\section{Affine invariant on planes}

\subsection{Standard region of a feature point}

Feature points or interest points can be defined as the points that have a large variation in the gray levels around itself $[2,11]$. These points have some uncertainty whether they are extracted by an interest point detector $[2,11]$ or by hand. Let $(\bar{x}, \bar{y})$ be the true position of a feature point $(x, y)$. If the errors $\Delta x=x-\bar{x}$ and $\Delta y=y-\bar{y}$ are regarded as random variables, their covariance matrix is written as

$$
\mathbf{V}=\sigma^{2} \mathbf{V}^{0}=\left(\begin{array}{cc}
E\left[\Delta x^{2}\right] & E[\Delta x \Delta y] \\
E[\Delta y \Delta x] & E\left[\Delta y^{2}\right]
\end{array}\right)
$$

where $E[\cdot]$ denotes expectation. Here, $\sigma$ indicates the absolute magnitude of the error and $\mathbf{V}^{0}$ indicates the geometric characteristics of the error, such as uniformity and isotropy. We call $\sigma$ the noise level and $\mathbf{V}^{0}$ the normalized covariance matrix. This normalized covariance matrix is computed as follows [4].

Let $I(i, j)$ be the gray level of pixel $(i, j)$. We evaluate the residual of self-matching in the form

$$
J(x, y)=\frac{1}{2} \sum_{(i, j) \in \mathcal{N}_{p}} w_{i j}(I(i+x, j+y)-I(i, j))^{2},
$$

where $x$ and $y$ are real numbers and $w_{i j}$ is an appropriate (maybe Gaussian) weight. $\mathcal{N}_{p}$ is a square neighborhood centered on the point $p$. We approximate Eq. (2) by a quadratic function over a neighborhood $\mathcal{X}$ of the origin $(0,0)$ in the form

$$
g(x, y)=\frac{1}{2}\left(n_{1} x^{2}+2 n_{2} x y+n_{3} y^{2}\right)=\frac{1}{2}\left(\begin{array}{ll}
x & y
\end{array}\right) \mathbf{H}_{e}\left(\begin{array}{l}
x \\
y
\end{array}\right),
$$

where the matrix $\mathbf{H}_{e}$ is the Hessian defined by

$$
\mathbf{H}_{e}=\left(\begin{array}{ll}
n_{1} & n_{2} \\
n_{2} & n_{3}
\end{array}\right)
$$

The coefficients $n_{1}, n_{2}$, and $n_{3}$ of $\mathbf{H}_{e}$ are determined by least squares [4]:

$$
\iint_{\mathcal{X}} w(x, y)(J(x, y)-g(x, y))^{2} d x d y \rightarrow \min
$$

Here, we use the Gaussian weight function $w(x, y)=\exp \left(-\left(x^{2}+y^{2}\right) / \sigma^{2}\right)$.

By resolving the minimization (5), we can obtain the coefficients $\left(n_{1}, n_{2}, n_{3}\right)$ of the Hessian $\mathbf{H}_{e}$. Then, the normalized covariance matrix is obtained by the following equation [4].

$$
\mathbf{V}^{0}=\mathbf{H}_{e}^{-1}
$$




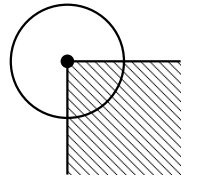

(a)

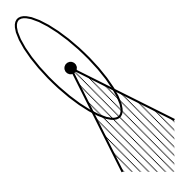

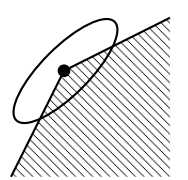

(c)

Figure 1: Covariance matrices of feature points

By using the standard region [3], we can illustrate the covariance matrices in the image. For example, the standard regions of three types of corner points are drawn by the ellipses in Figure 1. Here, we can regard the ellipses as images of a corner on a plane from three different view points: (a) from the facade of the plane, (b) from the lowerleft of the plane, and (c) from the lower-right of the plane. Thus, if we normalize the standard regions with an adequate scale and rotation, we can regard the gray levels in the normalized standard region as an affine invariant on a plane $[8,9]$.

\subsection{Gray level profile between two feature points}

We consider two feature points on a plane in a scene. If we project the plane into image planes of two cameras, the gray level profile between the two projected points in each image is an affine invariant $[1,12]$. If there are three points on the plane, the gray levels in the triangular region constructed by the three points are also affine invariant [10].

\section{Triplet vector descriptor and normalized triangular region vector}

Here, we propose the triplet vector descriptor for matching images. It is an affine invariant and consists of one profile vector and two normalized standard region vectors. The profile vector is defined by the gray level or color profile between two feature points in an image. The normalized standard region vector is defined by the gray levels inside the normalized standard region. In order to compute similarities between two descriptors simply, we fix the sizes of the vectors by sampling pixels from the line segment or the region.

\subsection{Profile vector}

Let $P(x, y)$ and $Q\left(x^{\prime}, y^{\prime}\right)$ be feature points in the image. Let $P_{i}\left(x_{i}, y_{i}\right)$ is the point obtained by dividing the line segment $\overline{P Q}$ into $N$ equal lengths, i.e. $P_{0}=P$ and $P_{N}=Q$. We define the profile vector $\mathbf{p}_{P Q}$ by

$$
\mathbf{p}_{P Q}=\left(\tilde{I}\left(x_{0}, y_{0}\right), \tilde{I}\left(x_{1}, y_{1}\right), \ldots, \tilde{I}\left(x_{N}, y_{N}\right)\right)^{\top}
$$

where

$$
\tilde{I}\left(x_{i}, y_{i}\right)=\sum_{(k, l) \in \mathcal{N}_{P_{i}}} \frac{1}{2 \pi \sigma^{2}} \exp \left(-\frac{k^{2}+l^{2}}{2 \sigma^{2}}\right) I(x+k, y+l) .
$$

Here, $I(x, y)$ is a gray level of the pixel $P(x, y)$ and $\mathcal{N}_{P_{i}}$ is a square neighborhood centered on the point $P_{i}$ for Gaussian smoothing. 


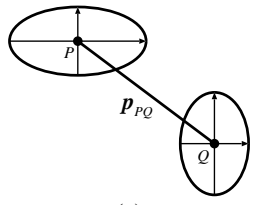

(a)

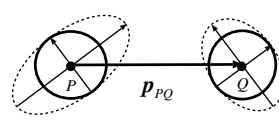

(b)

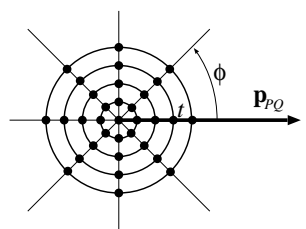

Figure 2: (a) Standard region. (b) Normalized Figure 3: Normalized standard region standard region (solid line).

\subsection{Normalized standard region vector}

Generally, the standard regions are ellipses, so we cannot compare the standard regions without normalization. Therefore, we transform the ellipses into the circles with an adequate rotation and scaling. However, we cannot determine the angle of the rotation. Here, we use the vector from the feature point to the other feature point as the reference axis of rotation (Fig.2).

Let $\mathbf{V}_{P}^{0}$ and $\mathbf{V}_{Q}^{0}$ be the normalized covariance matrices of the feature points $P$ and $Q$ respectively. As illustrated in Figure 3, we define the normalized standard region vector with respect to the point $P$ by sampling inside of the normalized circle:

$$
\begin{gathered}
\mathbf{r}_{P}=(\tilde{I}(\mathbf{x}(0,0)), \ldots, \tilde{I}(\mathbf{x}(L t, 0)), \tilde{I}(\mathbf{x}(0, \phi)), \ldots, \tilde{I}(\mathbf{x}(L t, \phi)), \\
\left.\quad \ldots, \tilde{I}\left(\mathbf{x}_{(0, M \phi)}\right), \ldots, \tilde{I}\left(\mathbf{x}_{(L t, M \phi)}\right)\right)^{\top}
\end{gathered}
$$

where,

$$
\begin{gathered}
\mathbf{x}(s, \theta)=\left(\begin{array}{l}
x \\
y
\end{array}\right)+s \mathbf{T}^{\top} \mathbf{S T R}\left(\begin{array}{c}
\cos \theta \\
\sin \theta
\end{array}\right), \quad s=0, \ldots, L t, \quad \theta=0, \ldots,(M-1) \phi, \\
t=\frac{r}{L}, \quad \phi=\frac{2 \pi}{M},
\end{gathered}
$$

where $r$ is the radius of the circle, $L$ and $M$ is the numbers of divisions of the radius and the angle, respectively. The matrices $\mathbf{T}$ and $\mathbf{S}$ are obtained by the spectral decomposition

$$
\mathbf{V}=\mathbf{T S} \mathbf{T}^{\top}=\left(\mathbf{t}_{1}, \mathbf{t}_{2}\right)\left(\begin{array}{cc}
\lambda_{1} & 0 \\
0 & \lambda_{2}
\end{array}\right)\left(\mathbf{t}_{1}, \mathbf{t}_{2}\right)^{\top}
$$

where $\lambda_{1}$ and $\lambda_{2}$ are the eigenvalues of the matrix $\mathbf{V}$ and $\mathbf{t}_{1}$ and $\mathbf{t}_{2}$ are their corresponding eigen vectors respectively. The matrix $\mathbf{R}$ indicates the rotation between the profile vector $\mathbf{p}_{P Q}$ and the horizontal axis.

\subsection{Triplet vector descriptor}

Now, we define the triplet vector descriptor $T_{P Q}$ with respect to the line segment defined by the two feature points $P$ and $Q$ in the image by

$$
T_{P Q}=\left\{\mathbf{p}_{P Q}, \mathbf{r}_{P}, \mathbf{r}_{Q}\right\} \text {. }
$$






Figure 4: Normalized triangular region vector $(K=3)$.

This triplet vector descriptor is an affine invariant on a plane. It is very powerful because it is constructed from two different affine invariants. In addition, we can easily compute the similarities between two triplet vector descriptor by computing SSD or correlations of each vector element.

\subsection{Normalized triangular region vector}

The triplet vector descriptor is powerful, but, unfortunately, the standard regions are sensitive to the positions of the feature points. Then, some wrong matches have high similarities on the triplet vector descriptors. In order to verify the similarities on the triplet vector descriptors, we check the similarities by another invariant on planes, which is called the normalized triangular region vector. We define the normalized triangular region vector by three pairs of triplet vector descriptor with high similarities.

Let $P, Q$, and $R$ be feature points in an image. The normalized triangular region vector is defined by

$$
\begin{aligned}
\mathbf{t}_{P Q R}=\left(\tilde{I}\left(\mathbf{x}^{\prime}(1,1)\right), \ldots, \tilde{I}\left(\mathbf{x}^{\prime}(1, K)\right), \tilde{I}\left(\mathbf{x}^{\prime}(2,1)\right), \ldots, \tilde{I}\left(\mathbf{x}^{\prime}(2, K-1)\right),\right. \\
\left.\quad \ldots, \tilde{I}\left(\mathbf{x}^{\prime}(K, 1)\right)\right)^{\top},
\end{aligned}
$$

where

$$
\mathbf{x}^{\prime}(i, j)=\mathbf{A}^{-1}\left(\begin{array}{c}
\frac{i}{K+2} \\
\frac{j}{K+2}
\end{array}\right), \quad i=1, \ldots, K, \quad j=1, \cdots, K-i+1 .
$$

Here, $\mathbf{A}$ is affine transformation from the original triangle in the image to the normalized triangle.

If there are three candidate pairs $\left\{P, P^{\prime}\right\},\left\{Q, Q^{\prime}\right\},\left\{R, R^{\prime}\right\}$ and the three pairs of the triplet vector descriptors $\left\{T_{P Q}, T_{P^{\prime} Q^{\prime}}\right\},\left\{T_{Q R}, T_{Q^{\prime} R^{\prime}}\right\},\left\{T_{R P}, T_{R^{\prime} P^{\prime}}\right\}$ have high similarities, we verify the three pairs by comparing the normalized triangular region vectors $\mathbf{t}_{P Q R}$ with $\mathbf{t}_{P^{\prime} Q^{\prime} R^{\prime}}$.

\section{Matching procedure}

In order to establish correspondences with the triplet vector descriptors and the normalized triangle region vectors, we adopt an voting table with respect to feature points in two images. We first vote the similarities between the triplet vector descriptors into the voting 
table, we next choose the correspondences that have high similarities. Finally, we check the remained correspondences by voting the similarities of the normalized triangle region vectors. We show the details of the procedure of the proposed method in the followings.

First, we detect feature points in each images by an interest point detector, such as Harris operator [2]. Let $P_{i}, i=1, \ldots, N$ be the feature points in the image $I$, and $Q_{j}, j=1$, $\ldots, N^{\prime}$ be the feature points in the image $I^{\prime}$.

1. Initialize the $N \times N^{\prime}$ voting table, which rows indicate $P_{i}$ and columns indicate $Q_{j}$, by zero.

2. Compute the covariance matrices for all $P_{i}$ and $Q_{j}$, individually. Then, normalize the scales of all the covariance matrices by dividing the average of the scale of the covariance matrices of all $P_{i}$.

3. Compute the profile vector $\mathbf{p}_{P_{\alpha} P_{\beta}}$ and the two normalized standard region vectors $\mathbf{r}_{P_{\alpha}}$ and $\mathbf{r}_{P_{\beta}}$ when $d_{\text {min }} \leq \operatorname{dist}\left(P_{\alpha}, P_{\beta}\right) \leq d_{\text {max }}$. Here, $\operatorname{dist}(P, Q)$ is the distance between $P$ and $Q$. From them, compose the triplet vector descriptors $T_{P_{\alpha} P_{\beta}}=$ $\left\{\mathbf{p}_{P_{\alpha} P_{\beta}}, \mathbf{r}_{P_{\alpha}}, \mathbf{r}_{P_{\beta}}\right\}$.

4. Pick pairs of the triplet vector descriptors that have high similarities and vote the similarities to the voting table.

5. If three pairs of triplet vector descriptors make triangles in each images, compute the normalized triangle region vector of the triangle and vote the similarities between the vectors into the corresponding feature points in the voting table.

6. Detect one-to-one correspondences from the voting table by finding the maximum bin in each row and each column.

7. Do RANSAC for choosing the correspondences that satisfy the epipolar constraint.

\section{Real image examples}

Figure 5 shows an example of a texture with repetitive pattern. Figure 5 (a) shows a stereo image pair of the scene. Figure 5 (b), (c), and (d) show the results obtained by the method of Kanazawa and Kanatani [5] ${ }^{1}$, the method of Lowe [6] ${ }^{2}$, and the proposed method, respectively. In Lowe's method, we do RANSAC with the epipolar constraint to the matches obtained by his program. In each figures, the left figure shows the matches obtained by each method and the right figure shows the 3-D reconstructions of the obtained matches.

Here, in order to detect feature points in an image, Kanazawa and Kanatani's method and the proposed method use Harris operator, but Lowe's method use SIFT operator[6]. So, the number of the detected feature points and their positions in the Lowe's results are different from the other results.

In this case, we can also see the characteristics of each method. The matches detected by Lowe's method are concentrate near the dirty marks of the wall, because the keypoint descriptors are almost the same in the repetitive texture region. In the results by Kanazawa and Kantani's method, there is no matches near the corner of the walls, because they use

\footnotetext{
${ }^{1}$ We use the program http://www.img.tutkie.tut.ac.jp/programs/index-e.html.

${ }^{2}$ We use the program http://www.cs.ubc.ca/ lowe/keypoints/.
} 


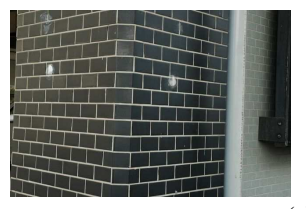

(a)

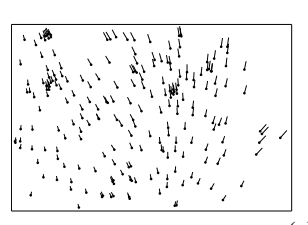

(c)
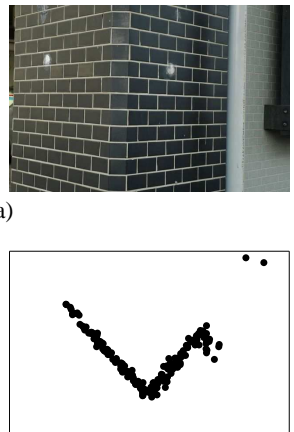



(b)

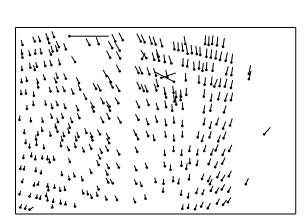

(d)

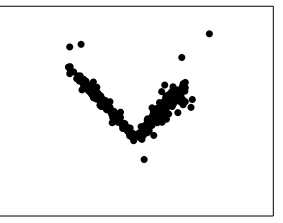

Figure 5: A scene includes a texture with repetitive pattern. (a) Stereo image pair. (b) Matches obtained by Kanazawa and Kanatani's method and their 3-D reconstructions (top view). (c) Matches obtained by Lowe's method and their 3-D reconstructions (top view). (d) Matches obtained by the proposed method and their 3-D reconstructions (top view).
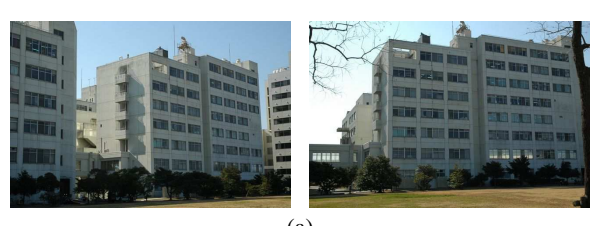

(a)

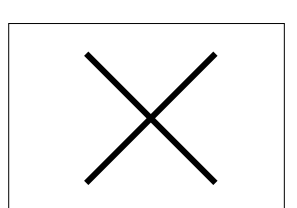

(c)

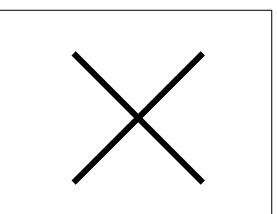

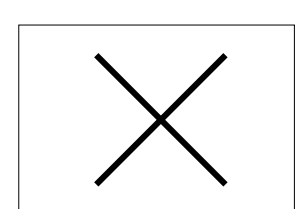

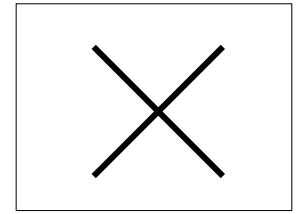

(b)

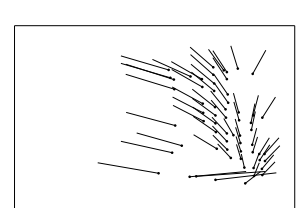

(d)

Figure 6: A wide baseline case. (a) Stereo image pair. (b) Results by Kanazawa and Kanatani's method. (c) Results by Lowe's method. (d) Matches by the proposed method and their 3-D reconstructions (top view).

the measure as matching with respect to global homography between images and their values are low in such the region. In contrast with these results, the matches are uniformly distributed in the images in our result.

Figure 6 shows an example of a building scene. Figure 6 (a) shows a stereo image pair of the scene. We can see this is a wide baseline case. Figure 6 (b), (c), and (d) show the results obtained by Kanazawa and Kanatani's method, Lowe's method, and the proposed method. In this figure, when we could not obtain any matches or 3-D reconstructions, we draw ' $\mathrm{X}$ ' mark in the figure. In this case, we cannot obtain the results by the Kanazawa and Kanatani's method and Lowe's method. By the proposed method, however, we can see we can obtain correct matches and their 3-D reconstructions are accurate.

Figure 7 shows an example of rotated images. Figure 7 (a) shows an stereo image pair and Figure 7 (b) shows the results obtained by Kanazawa and Kanatani's method. We see 


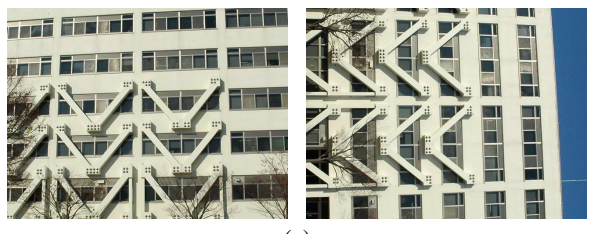

(a)
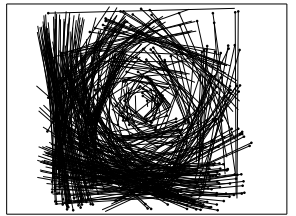

(c)

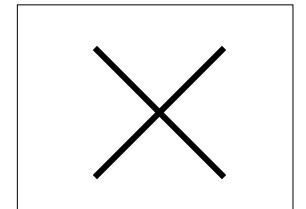

(b)



(d)
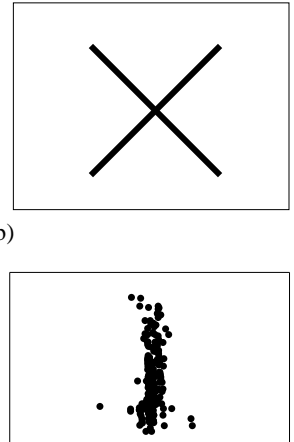

Figure 7: Image rotation. (a) Stereo image pair. (b) Results by Kanazawa and Kanatani's method. (c) Matches by Lowe's method and their 3-D reconstructions (side view). (d) Matches by the proposed method and their 3-D reconstructions (side view).

Table 1: The number of matches and their elapsed times.

\begin{tabular}{c||r|r|r|r|r|r}
\hline \multicolumn{1}{c||}{ Kanazawa and Kanatani's method } & \multicolumn{2}{c|}{ Lowe's method } & \multicolumn{2}{c}{ Proposed method } \\
\cline { 2 - 7 } & \# of matches & time (sec) & \# of matches & time (sec) & \# of matches & time (sec) \\
\hline Figure 5 & 235 & 16.64 & 217 & 7.05 & 330 & 20.49 \\
Figure 6 & - & - & - & - & 62 & 16.68 \\
Figure 7 & - & - & 465 & 14.43 & 294 & 16.23 \\
\hline
\end{tabular}

we could not obtain any matches and 3-D reconstructions by Kanazawa and Kanatani's method. Figure 7 (c) and (d) shows the results obtained by Lowe's method and the proposed method, respectively. We can see Lowe's method and the proposed method could detect many correct matches.

Figure 8 and Figure 9 show two examples of the images from the web page: "Affine Covariant Features ${ }^{3}$." In these examples, we omit the results by Kanazawa and Kanatani's method. We also omit the results using the two "Zoom+rotation" image packages because these two image packages are almost degenerated cases for the epipolar constraint. The results are shown in Table 2 . In Table $2, \# i-\# j$ means that we use the $i$-th image and the $j$-th image for a stereo image pair. We see the results obtained by Lowe's method are better than those by the proposed method. This is due to the fact that our program use standard Harris operator for detecting feature points and their detected positions may be different between two view points. We also see that the proposed method also detect correct matches but its number is smaller than that of the Lowe's method. On the other hand, we see the decreasing ratio of the number of the matches from "\#1-\#2" to "\#1-\#6" is smaller than that of the Lowe's method. It means if we use the other one instead of the Harris operator we can obtain the correct matches stably than these results. So, we need to replace the feature point detector in our program with an affine invariant detector like Harris-Affine [8].

In these experiments, we can see that the Kanazawa and Kanatani's method cannot handle wide baseline cases. We also see the Lowe's method is powerful but is a little weak

\footnotetext{
${ }^{3}$ We use the images from http://www. robots.ox.ac.uk/ vgg/research/affine/.
} 


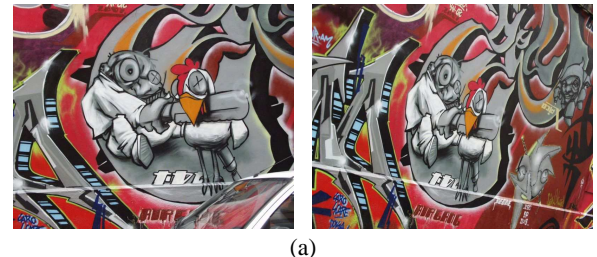

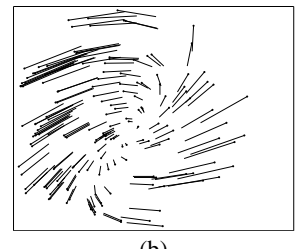



(c)

Figure 8: An example of "viewpoint1 (graf)" images. (a) Stereo image pair (img1.ppm and img3.ppm). (b) Matches by Lowe's method. (c) Matches by the proposed method.

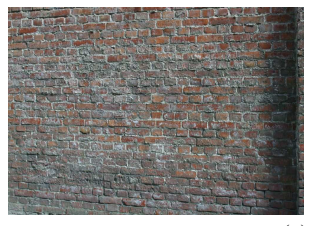

(a)

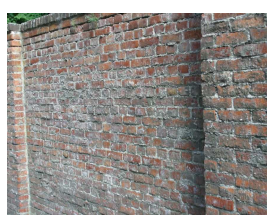



(b)

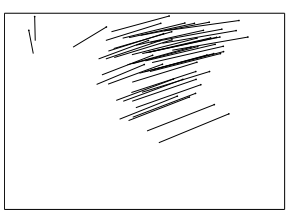

(c)

Figure 9: An example of "viewpoint2 (wall)" images. (a) Stereo image pair (img1.ppm and img5.ppm). (b) Matches by Lowe's method. (c) Matches by the proposed method.

in precise repetitive patterns (Figure 5) and simultaneous changes in scale and viewpoints (Figure 6) because keypoint descriptors are almost the same in such cases.

In all experiments in this paper, we used the size of the profile vector is 10 , the radius of the normalized standard region is 6 , the number of division in radius is 3 , and the number of the angle is 4 . We use $d_{\min }=10$ and $d_{\max }=60$. We used Pentium $4,3.2 \mathrm{GHz}$ for the CPU with $1 \mathrm{~GB}$ main memory and Fedora Core 4 for the OS. In Table 1, we show the number of matches and the elapsed times for computing in each results.

\section{Conclusion}

We have proposed an image matching method using triplet vector descriptor ${ }^{4}$. It consists of two types of affine invariants on planes. In order to establish correspondences, we vote the similarities between the descriptors into the voting table. After voting, we verify the matches with high similarities by another affine invariant normalized triangle region vectors. After enforcing the uniqueness of the candidate matches, we finally adopt RANSAC with the epipolar constraint for removing outliers. We show the effectiveness of our method by real image examples.

In future works, we replace the corner detector with the other one for detecting many correct matches and we also must reduce the computing time.

\section{Acknowledgement}

This work was supported in part by the Ministry of Education, Culture, Sports, Science and Technology, Japan, under the Grant for 21st Century COE Program "Intelligent Human Sensing."

\footnotetext{
${ }^{4}$ The source programs is available at the web page:

http://www.img.tutkie.tut.ac.jp/programs/index-e.html.
} 
Table 2: The number of matches of the results of "Affine Covariant Features."

\begin{tabular}{l||r|r|r|r|r||r|r|r|r|r}
\hline \multicolumn{1}{l||}{ Package } & \multicolumn{4}{|c||}{ Lowe's method } & \multicolumn{4}{|c}{ Proposed method } \\
\cline { 2 - 10 } & $\# 1-\# 2$ & $\# 1-\# 3$ & $\# 1-\# 4$ & $\# 1-\# 5$ & $\# 1-\# 6$ & $\# 1-\# 2$ & $\# 1-\# 3$ & $\# 1-\# 4$ & $\# 1-\# 5$ & $\# 1-\# 6$ \\
\hline "Blur 1" & 671 & 509 & 292 & 206 & 141 & 205 & 146 & 63 & 17 & 8 \\
"Blur 2" & 943 & 643 & 224 & 111 & 59 & 100 & 46 & 1 & 9 & 0 \\
"JPEG" & 2493 & 1777 & 931 & 415 & 177 & 396 & 352 & 279 & 201 & 70 \\
"Light" & 1017 & 785 & 568 & 483 & 291 & 294 & 179 & 61 & 27 & 0 \\
"Viewpoint 1" & 887 & 165 & 12 & 0 & 0 & 48 & 35 & 3 & 0 & 0 \\
"Viewpoint 2" & 4349 & 2607 & 739 & 63 & 0 & 291 & 226 & 134 & 45 & 0 \\
\hline
\end{tabular}

\section{References}

[1] F. Fraundorfer and H. Bischof. Affine invariant region matching using geometric hashing of line structures. In Proc. 27th Workshop of Austrian Assoc. for Patt. Recog. (ÖAGM/AAPR), pages 57-64, Laxenburg, Austria, 2003.

[2] C. Harris and M. Stephens. A combined corner and edge detector. In Proc. 4th Alvey Vision Conf., pages 147-151, Manchester, U.K., August 1988.

[3] K. Kanatani. Statistical Optimization for Geometric Computation: Theory and Practice. Elsevier Science, Amsterdam, 1996.

[4] Y. Kanazawa and K. Kanatani. Do we really have to consider covariance matrices for image features? Electronics and Communications in Japan, Part 3, 86(1):1-10, 2003.

[5] Y. Kanazawa and K. Kanatani. Robust image matching preserving global consistency. In Proc. 6th Asian Conf. Comput. Vision, pages 1128-1133, Jeju Island, Korea, January 2004.

[6] D.G. Lowe. Distinctive image features from scale-invariant keypoint. Int. J. Comput. Vision, 60(2):91-110, 2004.

[7] J. Matas, O. Chum, M. Urban, and T. Pajdla. Robust wide baseline stereo from maximally stable extremal regions. In Proc. 13th British Machine Vision Conf., pages 384-393, Cardiff, U.K., September 2002.

[8] K. Mikolajczyk and C. Schmid. Scale \& affine invariant interest point detector. Int. J. Comput. Vision, 60(1):63-86, 2004.

[9] K. Mikolajczyk, T. Tuytellaars, C. Schmid, Z. Zisserman, J. Matas, F. Schaffalitzky, K. Kadir, and L.V. Gool. A comparizon of affine region detectors. Int. J. Comput. Vision, online, 2006.

[10] F. Schaffalitzky and A. Zisserman. A viewpoint invariant texture matching and wide baseline stereo. In Proc. 8th Int. Conf. Comput. Vision, pages 636-643, Vancouver, Canada, July 2001.

[11] S.M. Smith and J.M. Brady. Susan - a new approach to low level image processing. Int. J. Comput. Vision, 23(1):45-78, 1997.

[12] D. Tell and S. Carlsson. Wide baseline point matching using affine invariants computed from intensity profiles. In Proc. 6th. European Conf. Comput. Vision, pages I-814-I-828, Dublin, Ireland, June/July 2000.

[13] T. Tuytelaars and L.V. Gool. Wide baseline stereo matching based on local, affinely invariant regions. In Proc. 11th British Machine Vision Conf., pages 412-422, Bristol, U.K., September 2000.

[14] Z. Zhang, R. Deriche, O. Faugeras, and Q.-T. Luong. A robust technique for matching two uncalibrated images through the recovery of the unknown epipolar geometry. Artif. Intell., 78:87-119, 1995. 